https://doi.org/10.15407/ujpe66.6.478

M.I. KOPP ${ }^{1}$ A.V. TUR, ${ }^{2}$ V.V. YANOVSKY ${ }^{1,3}$

${ }^{1}$ Institute for Single Crystals, Nat. Acad. of Sci. of Ukraine

(60, Nauky Ave., Kharkiv 61001, Ukraine)

2 Université de Toulouse [UPS], CNRS, Institut de Recherche en Astrophysique et Planétologie

(9, Ave. du Colonel Roche, BP 44346, 31028 Toulouse Cedex 4, France)

${ }^{3}$ V.N. Karazin Kharkiv National University

(4, Svobody Sq., Kharkiv 61022, Ukraine; e-mail: yanovsky@isc.kharkov.ua)

\title{
NONLINEAR VORTEX STRUCTURES DRIVEN BY SMALL-SCALE NONHELICAL FORCES IN OBLIQUELY ROTATING STRATIFIED FLUIDS
}

\begin{abstract}
We study a new type of large-scale instability in obliquely rotating stratified fluids with smallscale nonhelical turbulence. The small-scale turbulence is generated by the external force with zero helicity and low Reynolds number. The theory uses the method of multiscale asymptotic expansions. The nonlinear equations for large-scale motions are obtained in the third order of perturbation theory. We consider a linear instability and stationary nonlinear modes. Solutions in the form of nonlinear Beltrami waves and localized vortex structures such as kinks of a new type are obtained.

Keywords: equations of hydrodynamics in the Boussinesq approximation, Coriolis force, multiscale asymptotic expansions, small-scale nonhelical turbulence, $\alpha$-effect.
\end{abstract}

\section{Introduction}

The problem of generation of large-scale vortex motions and formation of stationary nonlinear vortex structures in turbulent media is very important. The characteristic scales of the velocity fields for these structures are much greater than the characteristic scales of turbulent motions or waves which engender their appearance. The study of the mechanism of generation of large-scale vortex structures (LSVS) by the small-scale turbulence has not only scientific interest, but also applied one. For instance, tornados, cyclones and anticyclones represent the particular cases of LSVS. They also play an important role in the dynamics of Earth's atmosphere, since they determine the global transport of air masses and are responsible

(c) M.I. KOPP, A.V. TUR, V.V. YANOVSKY, 2021 for the weather and climate on our planet [1-3]. The study of the mechanisms of LSVS generation is important for a number of astrophysical problems such as the origin of the Great Spot of Jupiter, Venus super rotation, vortex structures in solar prominences, and so on [4-8]. The generation of LSVS in atmospheres and bowels of the planets (or other space objects) is essentially due to thermal phenomena occurring under the influence of internal or external sources of the thermal energy. Articles [9-11] present the theory of convective vortex dynamos. It follows from this theory that the small-scale helical turbulence leads to a large-scale instability, which engenders the formation of one convective cell considered as a huge vortex of the tropical cyclone type. Several of the numerical [12] and laboratory [13] experiments confirm this. The theory of convective vortex dynamo was further developed in $[14,15]$ with the use of the method

ISSN 2071-0194. Ukr. J. Phys. 2021. Vol. 66, No. 6 
of multiscale asymptotic expansions. This method, unlike the functional techniques $[16,17]$ used in $[9$ 11] allows one to determine strictly the principal order of perturbation theory in which the instability arises. The method of multiscale asymptotic expansions to describe the generation of LSVS in reflective noninvariant turbulence was first used in [18]. The small Reynolds number is a parameter of the asymptotic expansions. The nonlinear stabilization of the convective large-scale instability discussed in $[14,15]$ leads to the formation of helical vortex solitons or kinks of a new type. The generation of a helical turbulence under natural conditions is usually associated with the influence of the Coriolis force on the turbulent motion $[19,20]$. Obviously, the question arises of the possibility of the generation of large-scale vortex fields in a rotating media under the influence of small-scale forces with zero helicity. An example of the generation of LSVS in a rotating incompressible fluid was found in [21]. It was also shown that the expansion of a large-scale instability in an inclined rotating fluid generates nonlinear large-scale helical vortex structures or localized Beltrami vortices within the internal helical structure.

In this paper, we give a generalization of the $\alpha$ effect found in [21] in the case of a temperaturestratified fluid. As a result of this generalization, we obtain the large-scale instability which generates the LSVS.

The organization of this paper is as follows. In Section 2, we obtain the averaged hydrodynamic equations in the Boussinesq approximation for an obliquely rotating fluid forming the large-scale fields using the method of multiscale asymptotic expansions. The technical aspect of this question is described in detail in Appendix I. The correlation functions in the averaged equations are expressed in terms of the small-scale fields in the zero-order approximation with respect to $R$. In Appendix II in order to obtain the averaged equations in the closed form, we find solutions for small-scale fields in the zero-order approximation. In Appendix III, we calculate the Reynolds stresses using these solutions. In Section 2, we obtain a closed system of nonlinear equations for large-scale vortex fields (vortex dynamo). In Section 3, we consider the generation of small large-scale vortex perturbations, which arise from an instability of the $\alpha$ effect type. The conditions of the appearance of this instability are determined depending on the effects of rotation and stratification of the medium. In Section 4 , an analytic and numerical analyses of the nonlinear equations in a steady state is performed, which shows the existence of nonlinear Beltrami waves and localized vortex structures in the form of kinks. The results obtained in the work can be applied to numerous geophysical and astrophysical problems.

\section{Equations for the Large-Scale Fields}

Let us consider a system of equations for perturbations of the velocity $\mathbf{v}$, temperature $T$, and pressure $P$ in the Boussinesq approximation with the constant temperature gradient $\nabla \bar{T}$ in the rotating coordinate system:

$$
\begin{aligned}
& \frac{\partial v_{i}}{\partial t}+v_{k} \frac{\partial v_{i}}{\partial x_{k}}=\nu \Delta v_{i}-\frac{1}{\bar{\rho}} \frac{\partial P}{\partial x_{i}}+2 \varepsilon_{i j k} v_{j} \Omega_{k}+g e_{i} \beta T+F_{0}^{i} \\
& \frac{\partial T}{\partial t}+v_{k} \frac{\partial T}{\partial x_{k}}-A e_{k} v_{k}=\chi \Delta T \\
& \frac{\partial v_{i}}{\partial x_{i}}=0
\end{aligned}
$$

The system of equations (1)-(3) describes the evolution of perturbations against the background of the basic equilibrium state $\bar{T}(z), \bar{P}(z)$, which is set by the constant temperature gradient $\nabla \bar{T}=-A \mathbf{e}$ $(A>0)$ (heated from below) and by the hydrostatic pressure gradient: $\nabla \bar{P}=\bar{\rho} \mathbf{g}-\bar{\rho}[\boldsymbol{\Omega} \times[\boldsymbol{\Omega} \times \mathbf{r}]]$, where $\mathbf{r}$ is the radius-vector of a fluid element. We consider the angular velocity of rotation $\boldsymbol{\Omega}$ as constant (solid rotation) and inclined to the plane $(X, Y)$, as shown in Fig. 1, i.e. for the Cartesian geometry problem: $\boldsymbol{\Omega}=\left(\Omega_{1}, \Omega_{2}, \Omega_{3}\right)$. Here, $\mathbf{e}=(0,0,1)$ is a unit vector in the direction of the axis $Z, \mathrm{~g}$ is the gravity directed vertically downward $\mathbf{g}=(0,0,-g)$, and $\beta$ is the coefficient of thermal expansion. Equation (1) includes the external force $\mathbf{F}_{0}$, which models the excitation source in the environment of small-scale and high-frequency fluctuations of the velocity field with small Reynolds number. Unlike the previous studies $[14,15]$, we consider here the nonhelical external force with the following properties:

$$
\begin{aligned}
& \operatorname{div} \mathbf{F}_{0}=0, \quad \mathbf{F}_{0} \operatorname{rot} \mathbf{F}_{0}=0, \quad \operatorname{rot} \mathbf{F}_{0} \neq 0 \\
& \mathbf{F}_{0}=f_{0} \mathbf{F}_{0}\left(\frac{x}{\lambda_{0}} ; \frac{t}{t_{0}}\right),
\end{aligned}
$$

where $\lambda_{0}$ is the characteristic scale, and $t_{0}$ is the characteristic time, and $f_{0}$ is the characteristic amplitude. The external force is given in the plane $(X, Y)$, 


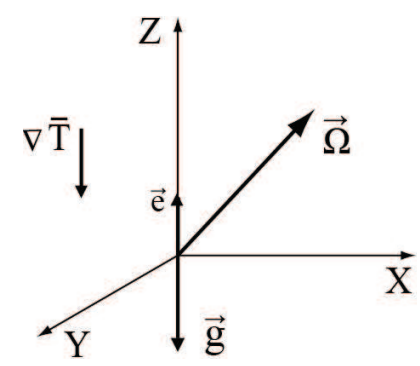

Fig. 1. In the general case, the angular velocity $\boldsymbol{\Omega}$ is inclined to the plane $(X, Y)$, where the external force $\mathbf{F}_{0 \perp}$ is located

where the perpendicular projection of the angular velocity is located. We choose the external force in the rotating coordinate system in the following form:

$F_{0}^{z}=0, \quad \mathbf{F}_{0 \perp}=f_{0}\left(\mathbf{i} \cos \phi_{2}+\mathbf{j} \cos \phi_{1}\right)$,

$\phi_{1}=\mathbf{k}_{1} \mathbf{x}-\omega_{0} t, \quad \phi_{2}=\mathbf{k}_{2} \mathbf{x}-\omega_{0} t$,

$\mathbf{k}_{1}=k_{0}(1,0,0), \quad \mathbf{k}_{2}=k_{0}(0,1,0)$.

Obviously, this nonhelical external force satisfies all conditions (4). In Eqs. (1)-(3), let us use the dimensionless variables. For convenience, we will designate these variables like dimensional ones:

$$
\begin{aligned}
& \mathbf{x} \rightarrow \frac{\mathbf{x}}{\lambda_{0}}, t \rightarrow \frac{t}{t_{0}}, \mathbf{v} \rightarrow \frac{\mathbf{v}}{v_{0}}, \mathbf{F}_{0} \rightarrow \frac{\mathbf{F}_{0}}{f_{0}}, P \rightarrow \frac{P}{\bar{\rho} p_{0}} \\
& t_{0}=\frac{\lambda_{0}^{2}}{\nu}, \quad p_{0}=\frac{v_{0} \nu}{\lambda_{0}}, \quad f_{0}=\frac{v_{0} \nu}{\lambda_{0}^{2}}, \quad T \rightarrow \frac{T}{\lambda_{0} A} .
\end{aligned}
$$

Then, in dimensionless variables, Eq. (1)-(3) takes the following form:

$$
\frac{\partial v_{i}}{\partial t}+R v_{k} \frac{\partial v_{i}}{\partial x_{k}}=\Delta v_{i}-\frac{\partial P}{\partial x_{i}}+\varepsilon_{i j k} v_{j} D_{k}+e_{i} \widetilde{\operatorname{Ra}} T+F_{0}^{i}
$$

$\frac{\partial T}{\partial t}+R v_{k} \frac{\partial T}{\partial x_{k}}-e_{k} v_{k}=\operatorname{Pr}^{-1} \Delta T$

$\frac{\partial v_{i}}{\partial x_{i}}=0$.

Here, $\widetilde{\mathrm{Ra}}=\frac{\mathrm{Ra}}{\mathrm{Pr}}, \mathrm{Ra}=\frac{g \beta A \lambda_{0}^{4}}{\nu \chi}$ is the Rayleigh number for the scale $\lambda_{0}, \operatorname{Pr}=\frac{\nu}{\chi}$ is the Prandtl number, and $D_{i}=\frac{2 \Omega_{i} \lambda_{0}^{2}}{\nu}$ is the dimensionless parameter of rotation for the scale $\lambda_{0}$ related to the Taylor number $\mathrm{Ta}_{i}=$ $=D_{i}^{2}$ which is the characteristic of the influence of the Coriolis force on viscous forces.

We will consider the Reynolds number $R=$ $=\frac{v_{0} t_{0}}{\lambda_{0}} \ll 1$ on the scale $\lambda_{0}$ as a small parameter of the asymptotic expansion. Concerning the parameters $D_{i}$ and $\mathrm{Ra}$, we do not choose any range of values for the moment. Let us examine the following formulation of the problem. We consider the external force as small and of high frequency. This force leads to small-scale fluctuations in the velocity and temperature fields against the equilibrium background. After the averaging, these quickly oscillating fluctuations vanish. Nevertheless, due to the small nonlinear interactions in some orders of perturbation theory, nonzero terms can occur after the averaging. This means that they are not oscillatory and are of largescale. From a formal point of view, these terms are secular, i.e., they create the conditions for the solvability of a large-scale asymptotic expansion. So, the purpose of this paper is to find and study the solvability equations, i.e., the equations for large-scale perturbations. The method of asymptotic equations is well presented in works [7], [14, 15]. In accordance with these papers, we introduce spatial and temporal derivatives in Eqs. (7)-(9) in the form of asymptotic expansions:

$$
\frac{\partial}{\partial t} \rightarrow \partial_{t}+R^{4} \partial_{T}, \quad \frac{\partial}{\partial x_{i}} \rightarrow \partial_{i}+R^{2} \nabla_{i}
$$

where $\partial_{i}$ and $\partial_{t}$ denote the derivatives with respect to fast variables $x_{0}=\left(\mathbf{x}_{0}, t_{0}\right)$ and $\nabla_{i}, \partial_{T}$ derivatives with respect to the slow variable $X=(\mathbf{X}, T)$. The variables $x_{0}$ and $X$ can be called small-scale and large-scale variables. To construct the nonlinear theory, the variables $\mathbf{v}, \mathbf{T}$, and $P$ are presented in the form of asymptotic series:

$$
\begin{aligned}
& \mathbf{v}(\mathbf{x}, t)=\frac{1}{R} \mathbf{W}_{-1}(X)+\mathbf{v}_{0}+R \mathbf{v}_{1}+ \\
& +R^{2} \mathbf{v}_{2}+R^{3} \mathbf{v}_{3}+\ldots, \\
& T(\mathbf{x}, t)=\frac{1}{R} T_{-1}(X)+T_{0}+R T_{1}+ \\
& +R^{2} T_{2}+R^{3} T_{3}+\ldots, \\
& P(\mathbf{x}, t)=\frac{1}{R^{3}} P_{-3}+\frac{1}{R^{2}} P_{-2}+\frac{1}{R} P_{-1}+P_{0}+ \\
& +R\left(P_{1}+\bar{P}_{1}(X)\right)+R^{2} P_{2}+R^{3} P_{3}+\ldots
\end{aligned}
$$

Substituting expansions (10)-(11) into the initial equations (7)-(9) and then gathering together the terms of the same order, we obtain the equations of the multiscale asymptotic expansion and write down

ISSN 2071-0194. Ukr. J. Phys. 2021. Vol. 66, No. 6 
the obtained equations up to the order $R^{3}$ including. The algebraic structure of the asymptotic expansion of Eqs. (7)-(9) in various orders of $R$ is given in Appendix I. It is also shown that in the order $R^{3}$ we get the main secular equation or equation for the large-scale fields:

$$
\begin{aligned}
& \partial_{T} W_{-1}^{i}-\Delta W_{-1}^{i}+\nabla_{k}\left(\overline{v_{0}^{k} v_{0}^{i}}\right)=-\nabla_{i} \bar{P}_{1}, \\
& \partial_{T} T_{-1}-\operatorname{Pr}^{-1} \Delta T_{-1}=-\nabla_{k}\left(\overline{v_{0}^{k} T_{0}}\right) .
\end{aligned}
$$

Equations (12)-(13) with secular equations are obtained in Appendix I:

$$
\begin{aligned}
& -\nabla_{i} P_{-3}+\widetilde{\operatorname{Ra}} e_{i} T_{-1}+\varepsilon_{i j k} W_{j} D_{k}=0 \\
& W_{-1}^{k} \nabla_{k} W_{-1}^{i}=-\nabla_{i} P_{-1} \\
& W_{-1}^{k} \nabla_{k} T_{-1}=0 \\
& \nabla_{i} W_{-1}^{i}=0 \\
& W_{-1}^{z}=0
\end{aligned}
$$

These equations are satisfied by choosing the following geometry for the velocity field:

$$
\begin{aligned}
& \mathbf{W}_{-1}=\left(W_{-1}^{x}(Z), W_{-1}^{y}(Z), 0\right), \\
& T_{-1}=T_{-1}(Z), \quad P_{-1}=\text { const. }
\end{aligned}
$$

In the frame of this quasi-two-dimensional approximation, we assume that the large-scale derivative over $Z$ is much larger than other derivatives, i.e.,

$$
\nabla_{Z} \equiv \frac{\partial}{\partial Z} \gg \frac{\partial}{\partial X}, \frac{\partial}{\partial Y}
$$

Then the system of equations (12)-(13) is simplified and takes the following form:

$$
\begin{aligned}
& \partial_{T} W_{1}-\nabla_{Z}^{2} W_{1}+\nabla_{Z}\left(\overline{v_{0}^{z} v_{0}^{x}}\right)=0, \quad W_{-1}^{x}=W_{1}, \\
& \partial_{T} W_{2}-\nabla_{Z}^{2} W_{2}+\nabla_{Z}\left(\overline{v_{0}^{z} v_{0}^{y}}\right)=0, \quad W_{-1}^{y}=W_{2}, \\
& \partial_{T} T_{-1}-P^{-1} \Delta T_{-1}+\nabla_{Z}\left(\overline{v_{0}^{z} T_{0}}\right)=0 .
\end{aligned}
$$

Equations (15)-(16) describe the evolution of largescale eddy fields W. In order to obtain the final closed form of Eqs. (15)-(16), we have to calculate the Reynolds stresses $\nabla_{k}\left(\overline{v_{0}^{k} v_{0}^{i}}\right)$. This shows that we need to find solutions for the small-scale velocity field $\mathbf{v}_{0}$. Appendix II contains a detailed technique to calculate the velocity field in a rotating stratified medium. Further, in Appendix III, the solutions for a small-scale velocity field $\mathbf{v}_{0}$ are used to find the Reynolds stresses. Then Eqs. (15)-(16) take the closed form:

$\left(\partial_{T}-\nabla_{Z}^{2}\right) \widetilde{W}_{1}=\frac{f_{0}^{2}}{2} D_{2} \nabla_{Z}\left(\alpha_{2}^{N L}\right)$,

$\left(\partial_{T}-\nabla_{Z}^{2}\right) \widetilde{W}_{2}=-\frac{f_{0}^{2}}{2} D_{1} \nabla_{Z}\left(\alpha_{1}^{N L}\right)$,

where

$\alpha_{1,2}^{N L}=\left(1+\widetilde{W}_{1,2}^{2}-\mathrm{Ra}\right)\left(1+\widetilde{W}_{1,2}^{2}\right)^{-1} r_{1,2}^{-1}$,

$r_{1,2}=\left(1+\widetilde{W}_{1,2}^{2}\right)^{2}+$

$+2\left(D_{1,2}^{2}-\mathrm{Ra}\right)\left(1-\widetilde{W}_{1,2}^{2}\right)+\left(D_{1,2}^{2}-\mathrm{Ra}\right)^{2}$.

To simplify the equations, we use here the designations: $W_{1}=1-W_{1}, \widetilde{W}_{2}=1-W_{2}$. Thus, in this section, we obtain the closed equations (18)-(19), which will be called the equations of nonlinear vortex dynamo in obliquely rotating stratified fluids with small-scale nonhelical force. If the rotation effect disappears $(\Omega=0)$, then the usual diffusion dissipation of large-scale fields occurs. In the limit of a homogeneous fluid, Eqs. (18) and (19) coincide with the results of [21]. First, we consider the stability of small perturbations of fields (linear theory) and then examine the question of the possible existence of stationary structures.

\section{Large-Scale Instability}

Equations (18)-(19) describe the nonlinear dynamics of large-scale disturbances of the vortex field $\mathbf{W}=\left(W_{1}, W_{2}\right)$. Therefore, it is of interest to clarify the question of the stability of small perturbations of the field $\mathbf{W}$. Then, for small values of $\left(W_{1}, W_{2}\right)$, Eqs. (18)-(19) are linearized and can be reduced to the following system of linear equations:

$\left\{\begin{array}{l}\partial_{T} W_{1}-\nabla_{Z}^{2} W_{1}-\alpha_{2} \nabla_{Z} W_{2}=0 \\ \partial_{T} W_{2}-\nabla_{Z}^{2} W_{2}+\alpha_{1} \nabla_{Z} W_{1}=0\end{array}\right.$

where we have introduced the following designations for the coefficients:

$$
\begin{aligned}
& \alpha_{1}=f_{0}^{2} D_{1} \times \\
& \times\left[\frac{\left(D_{1}^{2}-\mathrm{Ra}-2\right)(2-\mathrm{Ra})+\mathrm{Ra}\left(4+\left(D_{1}^{2}-\mathrm{Ra}\right)^{2}\right)}{\left(4+\left(D_{1}^{2}-\mathrm{Ra}\right)^{2}\right)^{2}}\right], \\
& \alpha_{2}=f_{0}^{2} D_{2} \times \\
& \times\left[\frac{\left(D_{2}^{2}-\mathrm{Ra}-2\right)(2-\mathrm{Ra})+\mathrm{Ra}\left(4+\left(D_{2}^{2}-\mathrm{Ra}\right)^{2}\right)}{\left(4+\left(D_{2}^{2}-\mathrm{Ra}\right)^{2}\right)^{2}}\right] .
\end{aligned}
$$




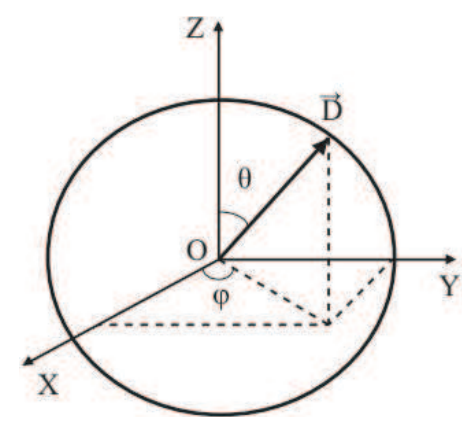

Fig. 2. Relation of Cartesian projections of the rotation parameter $\mathbf{D}$ (or angular velocity of rotation $\boldsymbol{\Omega}$ ) with their projections in the spherical coordinate system

It is clear that Eqs. (20) are similar to the equations for the vortex dynamo [9-15]. To study the large-scale instability described by the system of equations (20), we choose perturbations in the form of plane waves with wave vector $\mathbf{K} \| O Z$, i.e.

$W_{1,2}=A_{W_{1,2}} \exp (-i \omega t+i K Z)$.

Substituting (22) into the system of equations (20), we get the dispersion equation:

$\left(-i \omega+K^{2}\right)^{2}-\alpha_{1} \alpha_{2} K^{2}=0$.

From Eq. (23), we find the instability increment:

$\Gamma=\operatorname{Im} \omega= \pm \sqrt{\alpha_{1} \alpha_{2}} K-K^{2}$.

Solutions (24) show the existence of instabilities for large-scale vortical perturbations, when $\alpha_{1} \alpha_{2}>0$. If $\alpha_{1} \alpha_{2}<0$, damped oscillations arise with frequency $\omega_{0}=\sqrt{\alpha_{1} \alpha_{2}} K$ instead of the instabilities. The coefficients $\alpha_{1}, \alpha_{2}$ give a positive feedback loop between the components of the velocity. It should be noted that, in the linear theory, the coefficients $\alpha_{1}, \alpha_{2}$ do not depend on the amplitudes of the fields and depend only on the rotation parameters $D_{1,2}$, Rayleigh number Ra, and amplitude of the external force $f_{0}$. Let us analyze the dependence of these coefficients on the dimensionless parameters assuming, for simplicity, that the dimensionless amplitude of the external force $f_{0}$ is equal to $f_{0}=10$. In the coefficients $\alpha_{1}, \alpha_{2}$, instead of the Cartesian projections $D_{1}$ and $D_{2}$, it is convenient to use their projections in the spherical coordinate system $(D, \phi, \theta)$ (see Fig. 2). The coordinate surface $D=$ const is a sphere, $\theta$ is a latitude, $\theta \in[0, \pi], \phi$ is a longitude $\phi \in[0,2 \pi]$. We analyze the dependence of the amplification coefficients $\alpha_{1}, \alpha_{2}$ on the effects of rotation and stratification, assuming for simplicity that $D_{1}=D_{2}$, which corresponds to a fixed value of a longitude $\phi=\pi / 4+\pi n$, where $n=0,1,2, \ldots, k, k$ is an integer. In this case, the amplification coefficients of the vortex perturbations are, respectively, equal to:

$$
\begin{aligned}
& \alpha=\alpha_{1}=\alpha_{2}=f_{0}^{2} \sqrt{2} D \sin \theta \frac{a}{b}, \\
& a=4\left(D^{2} \sin ^{2} \theta-2 \mathrm{Ra}-4\right)(2-\mathrm{Ra})+ \\
& +\frac{\mathrm{Ra}}{2}\left(16+\left(D^{2} \sin ^{2} \theta-2 \mathrm{Ra}\right)^{2}\right), \\
& b=\left(16+\left(D^{2} \sin ^{2} \theta-2 \mathrm{Ra}\right)^{2}\right)^{2} .
\end{aligned}
$$

We can see from this equation that, at the poles $(\theta=0, \theta=\pi)$, the generation of vortex perturbations is not effective, because $\alpha \rightarrow 0$. The dependence of $\alpha$ coefficient on the stratification parameter of a fluid (Rayleigh number $\mathrm{Ra}$ ) at a fixed latitude $\theta=\pi / 2$, and the number $D=2$ is presented in the left part of Fig. 3. It shows the case of a homogeneous medium $\mathrm{Ra}=0$, where the generation of large-scale vortex perturbations is caused by the external nonhelical small-scale force and the Coriolis force [21]. Figure 3 shows that the presence of a temperature stratification $(\mathrm{Ra} \neq 0)$ can engender a significant increase in the coefficient $\alpha$. Consequently, we have a faster generation of large-scale vortex perturbations, than in a homogeneous medium. This effect manifests itself especially with the numbers $\mathrm{Ra} \rightarrow 2$. Further, with the increase in Rayleigh numbers, the coefficient $\alpha$ decreases. It is also interesting to find out the influence of the rotation effect on the amplification coefficients $\alpha$. For these purposes, we take the value of the Rayleigh number $\mathrm{Ra}=2$ at $\theta=\pi / 2$. In this case, the functional dependence $\alpha(D)$ is shown in the right part of Fig. 3. This shows that, for some parameter $D$, the coefficient $\alpha$ reaches its maximum value $\alpha_{\max }$. Then, as $D$ increases, the coefficient $\alpha$ tends gradually to zero, i.e. the suppression of the $\alpha$-effect occurs. A similar phenomenon was described in $[19,20]$. The left part of Fig. 4 shows the plot of the joint effect of rotation and stratification in the plane $(D, \mathrm{Ra})$. Here, the instability area is highlighted in gray. The maximum increment of instability $\Gamma_{\max }=\frac{\alpha_{1} \alpha_{2}}{4}$ is reached for the wave number $K_{\max }=\frac{\sqrt{\alpha_{1} \alpha_{2}}}{2}$. The plot of the function $\Gamma$ versus the wave number $K$ (see the right part of Fig. 4) has a standard form of the $\alpha$-effect. Thus, the development 

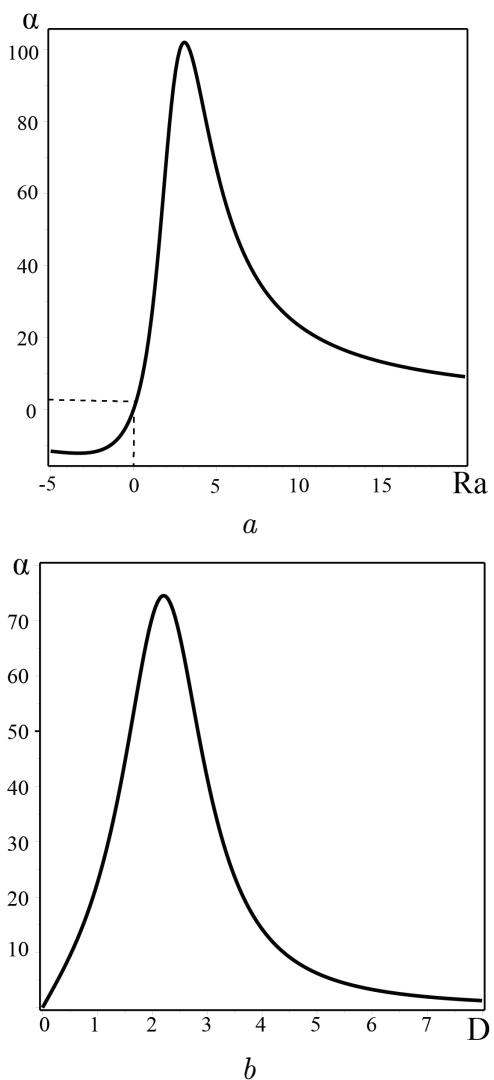

Fig. 3. The plot of the $\alpha$-effect of the parameter of stratification of a medium Ra (Rayleigh number) (a), the plot of the $\alpha$-effect of the parameter of rotation $D(b)$

of a large-scale instability in the rotating stratified atmosphere generates large-scale helical circularly polarized vortices of the Beltrami type.

\section{Stationary Nonlinear Vortex Structures}

It is obvious that, as the amplitude increases, the nonlinear terms decrease, and the instability becomes saturated. As a result, the nonlinear vortex structures appear. In order to find these structures, let us examine the stationary case of Eqs. (18)-(19) and intergrate once with respect to $Z$. For the sake of simplicity, we assume that $D_{1}=D_{2}$ and $\theta=\pi / 2$. Consequently, we get a system of nonlinear equations of the following form:

$$
\begin{aligned}
& \frac{d \widetilde{W}_{1}}{d Z}=-f_{0}^{2} D \sqrt{2}\left(1+\widetilde{W}_{2}^{2}-\mathrm{Ra}\right) \times \\
& \times\left(1+\widetilde{W}_{2}^{2}\right)^{-1} \widetilde{r}_{2}^{-1}+C_{1}, \\
& \frac{d \widetilde{W}_{2}}{d Z}=f_{0}^{2} D \sqrt{2}\left(1+\widetilde{W}_{1}^{2}-\mathrm{Ra}\right)\left(1+\widetilde{W}_{1}^{2}\right)^{-1} \widetilde{r}_{1}^{-1}+C_{2} .
\end{aligned}
$$
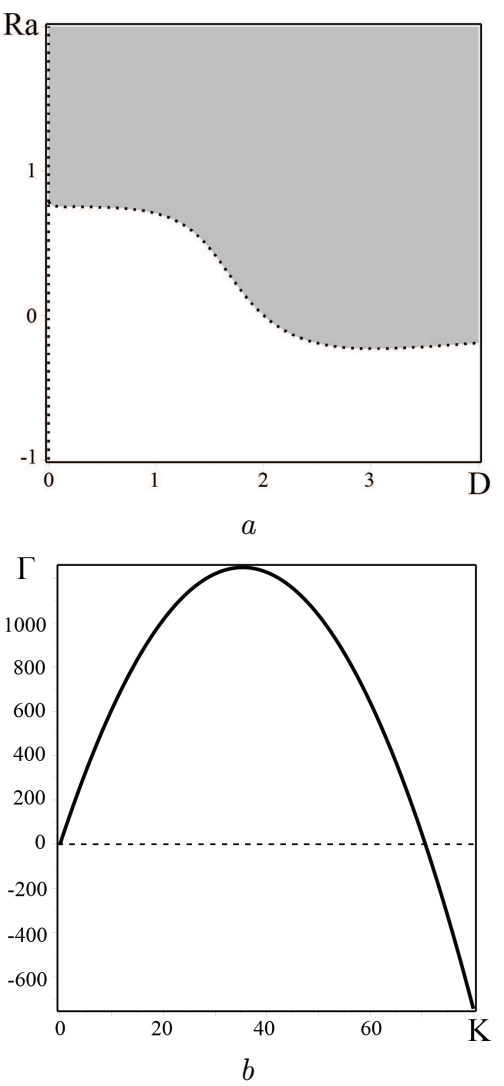

Fig. 4. The plot for $\alpha$ in the plane $(D, \mathrm{Ra})$, where the gray color shows the region corresponding to positive values of $\alpha$ (unstable solutions), and the white color - negative values of $\alpha(a)$; the plot of the dependence of the instability increment on the wave numbers $K$ for the parameters $D=2, \mathrm{Ra}=2(b)$

Here,

$\widetilde{r}_{1,2}=4\left(1+\widetilde{W}_{1,2}^{2}\right)^{2}+$

$+4\left(D^{2}-2 \mathrm{Ra}\right)\left(1-\widetilde{W}_{1,2}^{2}\right)+\left(D^{2}-2 \mathrm{Ra}\right)^{2}$.

$C_{1}, C_{2}$ are arbitrary constants of integration. It should be noted that the dynamic system of equations (25)-(26) is conservative and, hence, is a Hamiltonian one:

$\frac{d \widetilde{W}_{1}}{d Z}=-\frac{\partial H}{\partial \widetilde{W}_{2}}, \quad \frac{d \widetilde{W}_{2}}{d Z}=\frac{\partial H}{\partial \widetilde{W}_{1}}$,

where the Hamiltonian has the form:

$H=H_{1}\left(\widetilde{W}_{1}\right)+H_{2}\left(\widetilde{W}_{2}\right)+C_{2} \widetilde{W}_{1}-C_{1} \widetilde{W}_{2}$.

The functions $H_{1,2}$ are respectively equal to:

$H_{1,2}=f_{0}^{2} D \sqrt{2} \int \frac{\left(1+\widetilde{W}_{1,2}^{2}-\mathrm{Ra}\right) d \widetilde{W}_{1,2}}{\left(1+\widetilde{W}_{1,2}^{2}\right) \widetilde{r}_{1,2}}$. 


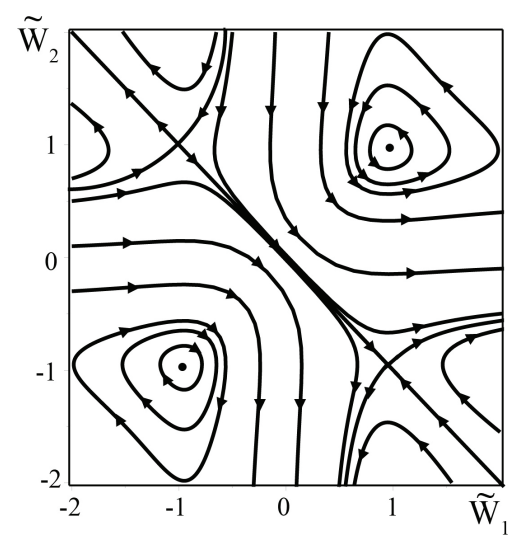

Fig. 5. Phase plane of the dynamical system of equations (25)-(26) with $C_{1}=-1$ and $C_{2}=1$. One can see the presence of closed trajectories around the elliptic points and separatrices which connect the hyperbolic points
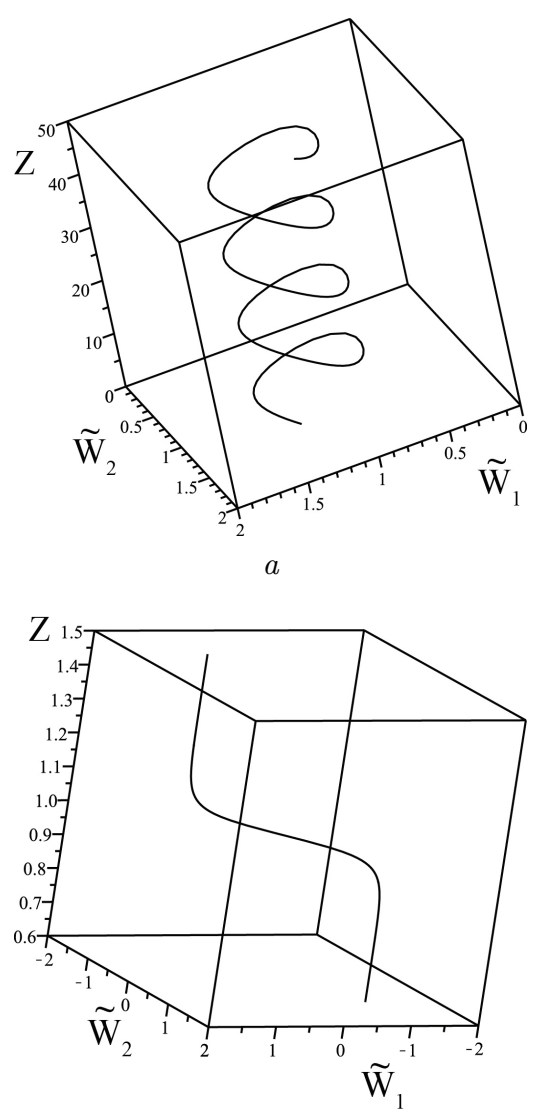

$b$

Fig. 6. A nonlinear helical wave which corresponds to a closed trajectory on the phase plane $(a)$; a localized nonlinear vortex structure (kink) which corresponds to the separatrix on the phase plane $\left(C_{1}=-1, C_{2}=1, D=\mathrm{Ra}=2\right)(b)$
Let us put $D=\mathrm{Ra}=2$ and $f_{0}=10$. Then we can calculate Hamiltonian (27):

$$
\begin{aligned}
& H=-\frac{25}{2} \sqrt{2}\left(\frac{\widetilde{W}_{1}\left(\widetilde{W}_{1}^{2}+3\right)}{\left(\widetilde{W}_{1}^{2}+1\right)^{2}}+\frac{\widetilde{W}_{2}\left(\widetilde{W}_{2}^{2}+3\right)}{\left(\widetilde{W}_{2}^{2}+1\right)^{2}}\right)- \\
& -\frac{25}{2} \sqrt{2}\left(\operatorname{arctg} \widetilde{W}_{1}+\operatorname{arctg} \widetilde{W}_{2}\right)+C_{2} \widetilde{W}_{1}-C_{1} \widetilde{W}_{2} .
\end{aligned}
$$

Since Eqs. (25)-(26) are Hamiltonian ones, only fixed points of two types, namely, elliptic and hyperbolic, can be observed in the phase space. This can be checked, if we carry out a qualitative analysis of the system of equations (25)-(26). Linearizing the righthand sides of Eqs. (25)-(26) in the neighborhood of fixed points, we establish their type and construct a phase portrait. As a result of the analysis, we find the appearance of four fixed points, two of the hyperbolic type and two of the elliptic one. The phase portrait of the dynamical system of equations (25)-(26) for the parameters $C_{1}=-1, C_{2}=1, D=\mathrm{Ra}=2$, and $f_{0}=10$ is shown in Fig. 5 . The phase portrait allows us to describe qualitatively the possible stationary solutions. The most interesting localized solutions correspond to the phase portrait trajectories, which connect the stationary (singular) points on the phase plane. Figure 5 presents closed trajectories on the phase plane around the elliptic points and separatrices which connect the hyperbolic points. The closed trajectories correspond to nonlinear periodic solutions or nonlinear waves. The separatrices correspond to localized vortex structures of the kink type (see Fig. 6).

\section{Conclusions}

In this paper, we have obtained a new type of largescale instability generated by the vertical temperature gradient and the small-scale force with zero helicity $\mathbf{F}_{0}$ rot $\mathbf{F}_{0}=0$ in an inclined rotating fluid. This force supports small-scale fluctuations in the fluid and models the effect of small-scale turbulence with the Reynolds number $R \ll 1$. It is assumed that the external force is in the plane $(X, Y)$. The force of gravity is directed vertically downward along the axis $O Z$. Using the method of multiscale asymptotic expansions, we get the closed system of equations for large-scale perturbations of the velocity. For small amplitudes, this system of equations describes the instability which is called the hydrodynamic $\alpha$ effect, since there is a positive feedback between the 
components of the velocity. It is shown that the instability arises only in the case where the angular velocity vector of rotation deviates from the axis $O Z$. The joint effect of rotation and stratification of the medium (heated from below) leads to a substantial enhancement of large-scale vortex perturbations, unlike the case of a homogeneous medium [21]. This phenomenon appears especially, when the parameters of the medium are $D \rightarrow 2$ and $\mathrm{Ra} \rightarrow 2$ (see Fig. 3). In this case, there is a maximum generation of small-scale helical motions due to the action of the Coriolis force and the inhomogeneity of the medium temperature. The rapid growth of vortex perturbations contributes to the increasing role of nonlinear effects and the consequent saturation of the instability. The study of the stationary state by numerical method with parameters $D=\mathrm{Ra}=2$ shows the existence of two types of solutions: nonlinear waves and kinks. These solutions are similar to those found in a homogeneous obliquely rotating fluid [21].

\section{APPENDIX I.}

\section{Multiscale asymptotic expansions}

Let us find the algebraic structure of the asymptotic expansion in various orders of $R$, starting from the lowest one. In the order $R^{-3}$, there is only one equation:

$\partial_{i} P_{-3}=0 \Rightarrow P_{-3}=P_{-3}(X)$.

In the order $R^{-2}$, we have the equations:

$\partial_{i} P_{-2}=0 \Rightarrow P_{-2}=P_{-2}(X)$.

In the order $R^{-1}$, we obtain a more complicated system of equations:

$\partial_{t} W_{-1}^{i}+W_{-1}^{k} \partial_{k} W_{-1}^{i}=-\partial_{i} P_{-1}-\nabla_{i} P_{-3}+\partial_{k}^{2} W_{-1}^{i}+$

$+\varepsilon_{i j k} W_{j} D_{k} e_{k}+\widetilde{\operatorname{Ra}} e_{i} T_{-1}$,

$\partial_{t} T_{-1}-\operatorname{Pr}^{-1} \partial_{k}^{2} T_{-1}=-W_{-1}^{k} \partial_{k} T_{-1}+W_{-1}^{z}$,

$\partial_{i} W_{-1}^{i}=0$.

The averaging of Eqs. (30)-(32) over the fast variables give the following secular equations:

$-\nabla_{i} P_{-3}+\widetilde{\operatorname{Ra}} e_{i} T_{-1}+\varepsilon_{i j k} W_{j} D_{k}=0$,

$W_{-1}^{z}=0$.

In the zero order in $R^{0}$, we have the equations:

$\partial_{t} v_{0}^{i}+W_{-1}^{k} \partial_{k} v_{0}^{i}+v_{0}^{k} \partial_{k} W_{-1}^{i}=-\partial_{i} P_{0}-\nabla_{i} P_{-2}+$

$+\partial_{k}^{2} v_{0}^{i}+\varepsilon_{i j k} v_{0}^{j} D_{k}+\widetilde{\operatorname{Ra}} e_{i} T_{0}+F_{0}^{i}$,

$\partial_{t} T_{0}-\operatorname{Pr}^{-1} \partial_{k}^{2} T_{0}=-W_{-1}^{k} \partial_{k} T_{0}-\partial_{k}\left(v_{0}^{k} T_{-1}\right)+v_{0}^{z}$,

$\partial_{i} v_{0}^{i}=0$.
These equations give one secular equation:

$\nabla P_{-2}=0 \quad \Rightarrow \quad P_{-2}=$ const.

Let us consider the equations in the first approximation in $R^{1}$ :

$\partial_{t} v_{1}^{i}+W_{-1}^{k} \partial_{k} v_{1}^{i}+v_{0}^{k} \partial_{k} v_{0}^{i}+v_{1}^{k} \partial_{k} W_{-1}^{i}+W_{-1}^{k} \nabla_{k} W_{-1}^{i}=$

$=-\nabla_{i} P_{-1}-\partial_{i}\left(P_{1}+\bar{P}_{1}\right)+\partial_{k}^{2} v_{1}^{i}+$

$+2 \partial_{k} \nabla_{k} W_{-1}^{i}+\operatorname{Ra} e_{i} T_{1}+\varepsilon_{i j k} v_{1}^{j} D_{k}$,

$\partial_{t} T_{1}-\operatorname{Pr}^{-1} \partial_{k}^{2} T_{1}-\operatorname{Pr}^{-1} 2 \partial_{k} \nabla_{k} T_{-1}=-W_{-1}^{k} \partial_{k} T_{1}-$

$-W_{-1}^{k} \nabla_{k} T_{-1}-v_{0}^{k} \partial_{k} T_{0}-v_{1}^{k} \partial_{k} T_{-1}+v_{1}^{z}$

$\partial_{i} v_{1}^{i}+\nabla_{i} W_{-1}^{i}=0$.

The secular equations follow from this system of equations:

$W_{-1}^{k} \nabla_{k} W_{-1}^{i}=-\nabla_{i} P_{-1}$,

$W_{-1}^{k} \nabla_{k} T_{-1}=0$,

$\nabla_{i} W_{-1}^{i}=0$.

The secular equations (42)-(44) are satisfied by choosing the following geometry:

$\mathbf{W}_{-1}=\left(W_{-1}^{x}(Z), W_{-1}^{y}(Z), 0\right)$,

$T_{-1}=T_{-1}(Z), P_{-1}=$ const.

In the second order in $R^{2}$, we obtain the equations:

$\partial_{t} v_{2}^{i}+W_{-1}^{k} \partial_{k} v_{2}^{i}+v_{0}^{k} \partial_{k} v_{1}^{i}+W_{-1}^{k} \nabla_{k} v_{0}^{i}+v_{0}^{k} \nabla_{k} W_{-1}^{i}+$

$+v_{1}^{k} \partial_{k} v_{0}^{i}+v_{2}^{k} \partial_{k} W_{-1}^{i}=-\nabla_{i} P_{2}-\nabla_{i} P_{0}+$

$+\partial_{k}^{2} v_{2}^{i}+2 \partial_{k} \nabla_{k} v_{0}^{i}+\widetilde{\operatorname{Ra}} e_{i} T_{2}+\varepsilon_{i j k} v_{2}^{j} D_{k}$

$\partial_{t} T_{2}-\operatorname{Pr}^{-1} \partial_{k}^{2} T_{2}-\operatorname{Pr}^{-1} 2 \partial_{k} \nabla_{k} T_{0}=-W_{-1}^{k} \partial_{k} T_{2}-$

$-W_{-1}^{k} \nabla_{k} T_{0}-v_{0}^{k} \partial_{k} T_{1}-v_{0}^{k} \nabla_{k} T_{-1}-$

$-v_{1}^{k} \partial_{k} T_{0}-v_{2}^{k} \partial_{k} T_{-1}+v_{2}^{z}$.

$\partial_{i} v_{2}^{i}+\nabla_{i} v_{0}^{i}=0$

It is easy to see that there are no secular terms in this order. Let us consider now the most important order $R^{3}$. In this order, we obtain the equations:

$\partial_{t} v_{3}^{i}+\partial_{T} W_{-1}^{i}+W_{-1}^{k} \partial_{k} v_{3}^{i}+v_{0}^{k} \partial_{k} v_{2}^{i}+W_{-1}^{k} \nabla_{k} v_{1}^{i}+$

$+v_{0}^{k} \nabla_{k} v_{0}^{i}+v_{1}^{k} \partial_{k} v_{1}^{i}+v_{1}^{k} \nabla_{k} W_{-1}^{i}+v_{2}^{k} \partial_{k} v_{0}^{i}+v_{3}^{k} \partial_{k} W_{-1}^{i}=$

$=-\partial_{i} P_{3}-\nabla_{i}\left(P_{1}+\bar{P}_{1}\right)+\partial_{k}^{2} v_{3}^{i}+2 \partial_{k} \nabla_{k} v_{1}^{i}+\Delta W_{-1}^{i}+$

$+\widetilde{\operatorname{Ra}} e_{i} T_{3}+\varepsilon_{i j k} v_{3}^{j} D_{k}$,

$\partial_{t} T_{3}+\partial_{T} T_{-1}-\operatorname{Pr}^{-1} \partial_{k}^{2} T_{3}-\operatorname{Pr}^{-1} 2 \partial_{k} \nabla_{k} T_{1}-$

$-\operatorname{Pr}^{-1} \Delta T_{-1}=-W_{-1}^{k} \partial_{k} T_{3}-W_{-1}^{k} \nabla_{k} T_{1}-$

$-v_{0}^{k} \partial_{k} T_{2}-v_{0}^{k} \nabla_{k} T_{0}-v_{1}^{k} \nabla_{k} T_{1}-v_{1}^{k} \nabla_{k} T_{-1}-$

$-v_{2}^{k} \partial_{k} T_{0}-v_{3}^{k} \partial_{k} T_{-1}+v_{3}^{z}$,

$\partial_{i} v_{3}^{i}+\nabla_{i} v_{1}^{i}=0$.

After the averaging, this system of equations with the fast variables, we obtain the main system of secular equations to describe the evolution of large-scale perturbations:

$\partial_{T} W_{-1}^{i}-\Delta W_{-1}^{i}+\nabla_{k}\left(\overline{v_{0}^{k} v_{0}^{i}}\right)=-\nabla_{i} \bar{P}_{1}$,

$\partial_{T} T_{-1}-\operatorname{Pr}^{-1} \Delta T_{-1}=-\nabla_{k}\left(\overline{v_{0}^{k} T_{0}}\right)$.

ISSN 2071-0194. Ukr. J. Phys. 2021. Vol. 66, No. 6 


\section{APPENDIX II.}

\section{Small-scale fields in the zero order in $R$}

In Appendix I, we obtain the equations in the zero order in $R$, which can be written in the following form:

$\widehat{D}_{W} v_{0}^{i}=-\partial_{i} P_{0}+\widetilde{\operatorname{Ra}} e_{i} T_{0}+\varepsilon_{i j k} v_{0}^{j} D_{k}+F_{0}^{i}$,

$\widehat{D}_{\theta} T_{0}=e_{k} v_{0}^{k}$,

$\partial_{i} v_{0}^{i}=0$

where we introduce the following designations for operators: $\widehat{D}_{W}=\partial_{t}-\partial_{k}^{2}+W_{-1}^{k} \partial_{k}, \widehat{D}_{\theta}=\partial_{t}-\operatorname{Pr}^{-1} \partial^{2}+W_{-1}^{k} \partial_{k}$.

Small-scale oscillations of the temperature are easily found from Eq. (55)

$T_{0}=\frac{v_{0}^{z}}{\widehat{D}_{\theta}}$.

Let us substitute (57) into (54). Using the condition of solenoidality of the fields $\mathbf{v}_{0}, \mathbf{F}_{0}$, we can find the pressure $P_{0}$ : $P_{0}=\widehat{P}_{1} u_{0}+\widehat{P}_{2} v_{0}+\widehat{P}_{3} w_{0}$.

Here, we introduced the following designations for operators:

$\widehat{P}_{1}=\frac{D_{2} \partial_{z}-D_{3} \partial_{y}}{\partial^{2}}, \quad \widehat{P}_{2}=\frac{D_{3} \partial_{x}-D_{1} \partial_{z}}{\partial^{2}}$,

$\widehat{P}_{3}=\frac{D_{1} \partial_{y}-D_{2} \partial_{x}}{\partial^{2}}+\widetilde{\operatorname{Ra}} \frac{\partial_{z}}{\widehat{D}_{\theta} \partial^{2}}$

and velocities: $v_{0}^{x}=u_{0}, v_{0}^{y}=v_{0}, v_{0}^{z}=w_{0}$. Using representation (58), we can eliminate the pressure from Eq. (54) and obtain the system of equations for the velocity fields in the zero approximation:

$\left(\widehat{D}_{W}+\widehat{p}_{1 x}\right) u_{0}+\left(\widehat{p}_{2 x}-D_{3}\right) v_{0}+\left(\widehat{p}_{3 x}+D_{2}\right) w_{0}=F_{0}^{x}$,

$\left(D_{3}+\widehat{p}_{1 y}\right) u_{0}+\left(\widehat{D}_{W}+\widehat{p}_{2 y}\right) v_{0}+\left(\widehat{p}_{3 y}-D_{1}\right) w_{0}=F_{0}^{y}$,

$\left(\widehat{p}_{1 z}-D_{2}\right) u_{0}+\left(\widehat{p}_{2 z}+D_{1}\right) v_{0}+$

$+\left(\widehat{D}_{W}-\frac{\widetilde{\mathrm{Ra}}}{\widehat{D}_{\theta}}+\widehat{p}_{3 z}\right) w_{0}=0$

The components of the tensor have the following form:

$\widehat{p}_{1 x}=\frac{D_{2} \partial_{x} \partial_{z}-D_{3} \partial_{x} \partial_{y}}{\partial^{2}}, \quad \widehat{p}_{2 x}=\frac{D_{3} \partial_{x}^{2}-D_{1} \partial_{x} \partial_{z}}{\partial^{2}}$,

$\widehat{p}_{3 x}=\frac{D_{1} \partial_{x} \partial_{y}-D_{2} \partial_{x}^{2}}{\partial^{2}}+\widetilde{\operatorname{Ra}} \frac{\partial_{x} \partial_{z}}{\widehat{D}_{\theta} \partial^{2}}$,

$\widehat{p}_{1 y}=\frac{D_{2} \partial_{y} \partial_{z}-D_{3} \partial_{y}^{2}}{\partial^{2}}, \quad \widehat{p}_{2 y}=\frac{D_{3} \partial_{y} \partial_{x}-D_{1} \partial_{y} \partial_{z}}{\partial^{2}}$,

$\widehat{p}_{3 y}=\frac{D_{1} \partial_{y}^{2}-D_{2} \partial_{y} \partial_{x}}{\partial^{2}}+\widetilde{\operatorname{Ra}} \frac{\partial_{y} \partial_{z}}{\widehat{D}_{\theta} \partial^{2}}$,

$\widehat{p}_{1 z}=\frac{D_{2} \partial_{z}^{2}-D_{3} \partial_{z} \partial_{y}}{\partial^{2}}, \quad \widehat{p}_{2 z}=\frac{D_{3} \partial_{z} \partial_{x}-D_{1} \partial_{z}^{2}}{\partial^{2}}$,

$\widehat{p}_{3 z}=\frac{D_{1} \partial_{z} \partial_{y}-D_{2} \partial_{z} \partial_{x}}{\partial^{2}}+\widetilde{\operatorname{Ra}} \frac{\partial_{z}^{2}}{\widehat{D}_{\theta} \partial^{2}}$.

The solution for system (59) can be found in accordance with Cramer's rule:

$u_{0}=\frac{1}{\Delta}\left(\widehat{d}_{1} F_{0}^{x}+\widehat{d}_{2} F_{0}^{y}\right)$,

486
$v_{0}=\frac{1}{\Delta}\left(\widehat{d}_{3} F_{0}^{x}+\widehat{d}_{4} F_{0}^{y}\right)$,

$w_{0}=\frac{1}{\Delta}\left(\widehat{d}_{5} F_{0}^{x}+\widehat{d}_{6} F_{0}^{y}\right)$,

where

$\widehat{d}_{1}=\left(\widehat{D}_{W}+\widehat{p}_{2 y}\right)\left(\widehat{D}_{W}-\frac{\widetilde{\mathrm{Ra}}}{\widehat{D}_{\theta}}+\widehat{p}_{3 z}\right)-$

$-\left(\widehat{p}_{2 z}+D_{1}\right)\left(\widehat{p}_{3 y}-D_{1}\right)$,

$\widehat{d}_{2}=\left(\widehat{p}_{3 x}+D_{2}\right)\left(\widehat{p}_{2 z}+D_{1}\right)-$

$-\left(\widehat{p}_{2 x}-D_{3}\right)\left(\widehat{D}_{W}-\frac{\widetilde{\mathrm{Ra}}}{\widehat{D}_{\theta}}+\widehat{p}_{3 z}\right)$

$\widehat{d}_{3}=\left(\widehat{p}_{3 y}-D_{1}\right)\left(\widehat{p}_{1 z}-D_{2}\right)-$

$-\left(D_{3}+\widehat{p}_{1 y}\right)\left(\widehat{D}_{W}-\frac{\widetilde{\mathrm{Ra}}}{\widehat{D}_{\theta}}+\widehat{p}_{3 z}\right)$

$\widehat{d}_{4}=\left(\widehat{D}_{W}+\widehat{p}_{1 x}\right)\left(\widehat{D}_{W}-\frac{\widetilde{\mathrm{Ra}}}{\widehat{D}_{\theta}}+\widehat{p}_{3 z}\right)-$

$-\left(\widehat{p}_{3 x}+D_{2}\right)\left(\widehat{p}_{1 z}-D_{2}\right)$,

$\widehat{d}_{5}=\left(D_{3}+\widehat{p}_{1 y}\right)\left(\widehat{p}_{2 z}+D_{1}\right)-\left(\widehat{D}_{W}+\widehat{p}_{2 y}\right)\left(\widehat{p}_{1 z}-D_{2}\right)$,

$\widehat{d}_{6}=\left(\widehat{p}_{2 x}-D_{3}\right)\left(\widehat{p}_{1 z}-D_{2}\right)-\left(\widehat{D}_{W}+\widehat{p}_{1 x}\right)\left(\widehat{p}_{2 z}+D_{1}\right)$.

Here, $\Delta$ is the determinant of the system of equations (59):

$\Delta=\left(\widehat{D}_{W}+\widehat{p}_{1 x}\right) \widehat{d}_{1}+\left(\widehat{p}_{2 x}-D_{3}\right) \widehat{d}_{3}+\left(\widehat{p}_{3 x}+D_{2}\right) \widehat{d}_{5}$.

In order to calculateexpressions (61)-(64), we present the external force (6) in complex form:

$\mathbf{F}_{0}=\mathbf{i} \frac{f_{0}}{2} e^{i \phi_{2}}+\mathbf{j} \frac{f_{0}}{2} e^{i \phi_{1}}+$ c.c.

Then all operators in formulae (61)-(64) act from the left on their eigenfunction. In particular:

$\widehat{D}_{W, H} e^{i \phi_{1}}=e^{i \phi_{1}} \widehat{D}_{W, \theta}\left(\mathbf{k}_{1},-\omega_{0}\right)$

$\widehat{D}_{W, \theta} e^{i \phi_{2}}=e^{i \phi_{2}} \widehat{D}_{W, \theta}\left(\mathbf{k}_{2},-\omega_{0}\right)$,

$\Delta e^{i \phi_{1}}=e^{i \phi_{1}} \Delta\left(\mathbf{k}_{1},-\omega_{0}\right)$,

$\Delta e^{i \phi_{2}}=e^{i \phi_{2}} \Delta\left(\mathbf{k}_{2},-\omega_{0}\right)$.

To simplify the formulae, we choose $k_{0}=1, \omega_{0}=1$ and introduce new designations:

$$
\begin{aligned}
& \widehat{D}_{W}\left(\mathbf{k}_{1},-\omega_{0}\right)=\widehat{D}_{W_{1}}^{*}=1-i\left(1-W_{1}\right), \\
& \widehat{D}_{W}\left(\mathbf{k}_{2},-\omega_{0}\right)=\widehat{D}_{W_{2}}^{*}=1-i\left(1-W_{2}\right), \\
& \widehat{D}_{\theta}\left(\mathbf{k}_{1},-\omega_{0}\right)=\widehat{D}_{\theta_{1}}^{*}=\operatorname{Pr}^{-1}-i\left(1-W_{1}\right), \\
& \widehat{D}_{\theta}\left(\mathbf{k}_{2},-\omega_{0}\right)=\widehat{D}_{\theta_{2}}^{*}=\operatorname{Pr}^{-1}-i\left(1-W_{2}\right) .
\end{aligned}
$$

Complex-conjugate quantities will be denoted by asterisk. When performing further calculations, a part of tensor components $\widehat{p}_{i j}\left(\mathbf{k}_{1},-\omega_{0}\right)$ and $\widehat{p}_{i j}\left(\mathbf{k}_{2},-\omega_{0}\right)$ vanishes. In view of this, the velocity field in the zero approximation is represented as

$u_{0}=\frac{f_{0}}{2} \frac{\widehat{A}_{2}^{*}}{\widehat{A}_{2}^{*} \widehat{D}_{W_{2}}^{*}+D_{2}^{2}} e^{i \phi_{2}}+$ c.c. $=u_{03}+u_{04}$,

ISSN 2071-0194. Ukr. J. Phys. 2021. Vol. 66, No. 6 
$v_{0}=\frac{f_{0}}{2} \frac{\widehat{A}_{1}^{*}}{\widehat{A}_{1}^{*} \widehat{D}_{W_{1}}^{*}+D_{1}^{2}} e^{i \phi_{1}}+$ c.c. $=v_{01}+v_{02}$,

$w_{0}=-\frac{f_{0}}{2} \frac{D_{1}}{\widehat{A}_{1}^{*} \widehat{D}_{W_{1}}^{*}+D_{1}^{2}} e^{i \phi_{1}}+\frac{f_{0}}{2} \frac{D_{2}}{\widehat{A}_{2}^{*} \widehat{D}_{W_{2}}^{*}+D_{2}^{2}} e^{i \phi_{2}}+$

+ c.c. $=w_{01}+w_{02}+w_{03}+w_{04}$,

where

$\widehat{A}_{1,2}^{*}=\widehat{D}_{W_{1,2}}^{*}-\frac{\widetilde{\mathrm{Ra}}}{\widehat{D}_{\theta_{1,2}}^{*}}$.

Components of the velocity satisfy the following relations:

$w_{02}=\left(w_{01}\right)^{*}, \quad w_{04}=\left(w_{03}\right)^{*}, \quad v_{02}=\left(v_{01}\right)^{*}$,

$v_{04}=\left(v_{03}\right)^{*}, \quad u_{02}=\left(u_{01}\right)^{*}, \quad u_{04}=\left(u_{03}\right)^{*}$.

\section{APPENDIX III.}

\section{Calculation of the Reynolds stresses}

To close Eqs. (15)-(16), we have to calculate the Reynolds stresses $T^{i k}=\overline{v_{0}^{i} v_{0}^{k}}$ or its components:

$T^{31}=\overline{w_{0} u_{0}}=\overline{w_{01}\left(u_{01}\right)^{*}}+\overline{\left(w_{01}\right)^{*} u_{01}}+$

$+\overline{w_{03}\left(u_{03}\right)^{*}}+\overline{\left(w_{03}\right)^{*} u_{03}}$,

$T^{32}=\overline{w_{0} v_{0}}=\overline{w_{01}\left(v_{01}\right)^{*}}+\overline{\left(w_{01}\right)^{*} v_{01}}+$

$+\overline{w_{03}\left(v_{03}\right)^{*}}+\overline{\left(w_{03}\right)^{*} v_{03}}$.

Substituting the solutions for the small-scale velocity fields (68)-(70) obtained in Appendix II into Eqs. (72)-(73), we can find the following expression for the correlators:

$T^{31}=\frac{f_{0}^{2}}{4} \frac{D_{2}\left(\widehat{A}_{2}+\widehat{A}_{2}^{*}\right)}{\left|\widehat{A}_{2} \widehat{D}_{W_{2}}+D_{2}^{2}\right|^{2}}$,

$T^{32}=-\frac{f_{0}^{2}}{4} \frac{D_{1}\left(\widehat{A}_{1}+\widehat{A}_{1}^{*}\right)}{\left|\widehat{A}_{1} \widehat{D}_{W_{1}}+D_{1}^{2}\right|^{2}}$.

Then, with the definition of operators (67) and (71), we write down the series of useful relations for the calculation of $T^{31}$ and $T^{32}$.

$$
\begin{aligned}
& \left|\widehat{D}_{W_{1,2}}\right|^{2}=\widehat{D}_{W_{1,2}} \hat{D}_{W_{1,2}}^{*}=1+\widetilde{W}_{1,2}^{2}, \\
& \left|\widehat{D}_{\theta_{1,2}}\right|^{2}=\widehat{D}_{\theta_{1,2}} \widehat{D}_{\theta_{1,2}}^{*}=\operatorname{Pr}^{-2}+\widetilde{W}_{1,2}^{2}, \\
& \left|\widehat{A}_{1,2}\right|^{2}=\hat{A}_{1,2} \widehat{A}_{1,2}^{*}=1+\widetilde{W}_{1,2}^{2}- \\
& -2 \operatorname{Ra} \frac{1-\operatorname{Pr} \widetilde{W}_{1,2}^{2}}{1+\operatorname{Pr}^{2} \widetilde{W}_{1,2}^{2}}+\frac{\operatorname{Ra}^{2}}{1+\operatorname{Pr}^{2} \widetilde{W}_{1,2}^{2}}, \\
& \widehat{D}_{W_{1,2}} \widehat{D}_{\theta_{1,2}}+\widehat{D}_{W_{1,2}}^{*} \widehat{D}_{\theta_{1,2}}^{*}=2\left(\operatorname{Pr}^{-1}-\tilde{W}_{1,2}^{2}\right), \\
& \widehat{D}_{W_{1,2}} \widehat{A}_{1,2}+\widehat{D}_{W_{1,2}}^{*} \widehat{A}_{1,2}^{*}= \\
& =2\left(1-\widetilde{W}_{1,2}^{2}\right)-2 \operatorname{Ra} \frac{1+\operatorname{Pr} \widetilde{W}_{1,2}^{2}}{1+\operatorname{Pr} \widetilde{W}_{1,2}^{2}} .
\end{aligned}
$$

ISSN 2071-0194. Ukr. J. Phys. 2021. Vol. 66, No. 6
Here, we apply the following designations: $\widetilde{W}_{1}=1-W_{1}$, $\widetilde{W}_{2}=1-W_{2}$. Using these relations, we can obtain the following expressions:

$$
\begin{aligned}
& \widehat{A}_{1,2}+\widehat{A}_{1,2}^{*}=2\left(1-\frac{\mathrm{Ra}}{1+\operatorname{Pr}^{2} \widetilde{W}_{1,2}^{2}}\right), \\
& \left|\widehat{D}_{W_{1,2}} \widehat{A}_{1,2}+D_{1,2}^{2}\right|^{2}=\left(1+\widetilde{W}_{1,2}^{2}\right)^{2}+ \\
& +\operatorname{Ra}^{2} \frac{1+\widetilde{W}_{1,2}^{2}}{1+\operatorname{Pr}^{2} \widetilde{W}_{1,2}^{2}}+2 D_{1,2}^{2}\left(1-\widetilde{W}_{1,2}^{2}\right)+D_{1,2}^{4}- \\
& -2 \operatorname{Ra} \frac{2+\widetilde{W}_{1,2}^{2}-\operatorname{Pr} \widetilde{W}_{1,2}^{4}-\left(1+\operatorname{Pr} \widetilde{W}_{1,2}^{2}\right)\left(1-D_{1,2}^{2}\right)}{1+\operatorname{Pr}^{2} \widetilde{W}_{1,2}^{2}} .
\end{aligned}
$$

Substituting (76) in (74)-(75), we can find an expressions for Reynolds stresses in the general form. For instance, for the atmosphere, the Prandtl number is approximately equal to one $\operatorname{Pr}=1$. In this case, the expressions for the components of Reynolds stresses are simplified:

$T^{31}=\frac{f_{0}^{2}}{2} D_{2} \frac{\left(1+\widetilde{W}_{2}^{2}-\mathrm{Ra}\right)}{\left(1+\widetilde{W}_{2}^{2}\right) r_{2}}$

$T^{32}=-\frac{f_{0}^{2}}{2} D_{1} \frac{\left(1+\widetilde{W}_{1}^{2}-\mathrm{Ra}\right)}{\left(1+\widetilde{W}_{1}^{2}\right) r_{1}}$,

where

$r_{1,2}=\left(1+\widetilde{W}_{1,2}^{2}\right)^{2}+2\left(D_{1,2}^{2}-\mathrm{Ra}\right)\left(1-\widetilde{W}_{1,2}^{2}\right)+\left(D_{1,2}^{2}-\mathrm{Ra}\right)^{2}$

1. A.S. Monin. Theoretical Fundamentals of Geophysical Hydrodynamics (Gidrometeoizdat, 1988) (in Russian).

2. O.G. Onishchenko, O.A. Pokhotelov, N.M. Astafieva. Generation of large-scale vortices and zonal winds in atmopheric planets. Physics-Uspekhi 178, 605 (2008).

3. B.Y. Shmerlin, M.V. Kalashnik. Convective instability of Rayleigh in the presence of phase transitions of the humidity. The formation of the large-scale eddies and cloud structures. Physics-Uspekhi 183, 497 (2013).

4. V.I. Petviashvili, O.A. Pokhotelov. Solitary Vortices in Plasma and Atmosphere (Energoatomizdat, 1989) (in Russian).

5. G.D. Aburjania. Self-Organization of Nonlinear Vortex Structures and Vortex Turbulence in Dispersive Media (Komkniga, 2006) (in Russian).

6. A.V. Kolesnichenko, M.Ya. Marov. Turbulence and SelfOrganization. Problems of Modeling Space and Natural Media (BINOM, 2009) (in Russian).

7. A. Tur, V. Yanovsky. Coherent Vortex Structures in Fluids and Plasmas (Springer, 2017) [ISBN: 978-3-319-52733-8].

8. A.M. Freedman, A.V. Khoperskov. Physics of Galactic Disks (FIZMATLIT, 2011) (in Russian).

9. S.S. Moiseev, P.B. Rutkevitch, A.V. Tur, V.V. Yanovsky. Vortex dynamos in a helical turbulent convection. Sov. Phys. JETP 67, 294 (1988).

10. E.A. Lypyan, A.A. Mazurov, P.B. Rutkevitch, A.V. Tur. Generation of large-scale vortices through the action of spiral turbulence of a convective nature. Sov. Phys.JETP $\mathbf{7 5}$, 838 (1992). 
11. S.S. Moiseev, K.R. Oganjan, P.B. Rutkevich, A.V. Tur, G.A. Khomenko, V.V. Yanovsky. Vortex dynamo in helical turbulence. In: Integrability and Kinetic Equations for Solitons (Naukova Dumka, 1990) (in Russian).

12. G.V. Levina, S.E. Startsev, V.D. Zimin, S.S. Moiseev. About another physical mechanism of large-scale structures generation at turbulent convection in the horizontal fluid layer. Phys. Chem. of the Earth Parts 21, 563 (1996).

13. G.V. Levina, S.S. Moiseev, P.B. Rutkevich. Hydrodynamic alpha-effect in a convective system. Advances in Fluid Mechanics 25, 111 (2000).

14. A.V. Tur, V.V. Yanovsky. Large-scale instability in hydrodynamics with stable temperature stratification driven by small-scale helical force. arXiv:1204.5024 v.1[physics. Fludyn.] (2012)

15. A.V. Tur, V.V. Yanovsky. Non linear vortex structure in stratified driven by small-scale helical forse. Open Journal of Fluid Dynamics 3, 64 (2013).

16. E.A. Novikov. Functionals and the random force method in turbulence theory. Sov. Phys. JETP 47 (11), 1919 (1964).

17. V.I. Klyatskin. Stochastic Equations and Waves in Randomly Inhomogeneous Media (Nauka, 1980) (in Russian).

18. U. Frisch, Z.S. She, P.L. Sulem. Large-scale flow driven by the anisotropic kinetic alpha effect. Physica D 28, 382 (1987).

19. G. Rüdiger. On the $\alpha$-effect for slow and fast rotation. Astron. Nachr. 299, 217 (1978).

20. G. Moffat. Magnetic Field Generation in Electrically Conducting Fluids (Cambridge University Press, 1978).
21. M.I. Kopp, A.V. Tur, V.V. Yanovsky. Nonlinear vortex structures in rotating fluid obliquely. Open Journal of Fluid Dynamics 5, 311 (2015).

Received 23.03.20

М.Й. Konn, A.В. Тур, В.В. Яновсъкий

НЕЛІНІЙНІ ВИХРОВІ СТРУКТУРИ,

ЯКІ ЗБУДЖУЮТЬСЯ ЗОВНІШНЬОЮ

НЕСПІРАЛЬНОЮ СИЛОЮ

В СТРАТИФІКОВАНІЙ РІДИНІ, ЩО ПОХИЛО ОБЕРТАЕТЬСЯ

Досліджується великомасштабна нестійкість в стратифікованій рідині, що похило обертається та є неспірально турбулентною. Турбулентність збуджується зовнішньою дрібномасштабною силою з нульовою спіральністю і малим числом Рейнольдса. Теорія побудована на основі методу багатомасштабних асимптотичних розкладів. Нелінійні рівняння для великомасштабних рухів отримано в третьому порядку теорії збурень. Досліджено лінійну нестійкість і стаціонарні нелінійні режими. Отримано розв'язки у вигляді нелінійних хвиль Бельтрамі та локалізованих вихрових структур-кінків нового типу.

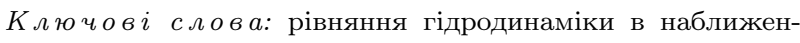
ні Буссінеска, сила Коріоліса, багатомасштабні асимптотичні розклади, дрібномасштабна спіральна турбулентність, $\alpha$-ефект. 